\title{
An adrenal tumour secreting multiple hormones
}

\author{
H N Rajaratnam ${ }^{1}$, S A Abhayaratna ${ }^{2}$, N P Somasundaram ${ }^{3}$, A Samarasekara $^{4}$, S K Kollure ${ }^{5}$ \\ Sri Lanka Journal of Diabetes, Endocrinology and Metabolism 2014; 4: 30-34
}

\begin{abstract}
A right homogeneous adrenal tumor was found incidentally, during abdominal computed tomography in a 72-year-old female patient, presenting with hypertension of 2 years duration. She had an elevated Aldosterone: Renin Ratio (ARR) and primary hyperaldosteronism was confirmed with a fludrocortisone suppression test. Plasma basal cortisol and adrenocorticotropic hormone (ACTH) levels were normal, but the plasma cortisol concentration could not be suppressed with dexamethasone. Therefore, an adrenal cortical adenoma with primary hyperaldosteronism and subclinical hypercortisolism was suspected. Urinary total metanephrines and vanillylmandelic acid (VMA) levels were also marginally elevated, indicating the possibility of a pheochromocytoma. After right adrenalectomy, the tumour was histologically demonstrated to be a pheochromocytoma, and the levels of all three hormone groups viz corticosteroid, mineralocorticoid and metanephrine levels normalized, indicating the possibility of a cosecretory small adrenocortical microadenoma.
\end{abstract}

Key words: cortical adenoma, pheochromocytoma, subclinical hypercortisolism

\section{Introduction}

Before the era of computed tomography (CT) and magnetic resonance imaging (MRI), adrenal tumours were identified by the detection of excessive hormone levels. Presently, due to technological advances and wide availability of imaging modalities, these tumours are often discovered incidentally during CT scanning or ultrasonography undertaken for other reasons and are called "incidentalomas". In most of the cases, clinical and biochemical features of excessive hormone levels are absent, but some of them demonstrate normal basal hormone levels with abnormal responses to dynamic testing. The prevalence of adrenal incidentalomas had been shown to be as high as $10 \%$ in older patients (1).

We describe a case of an elderly woman with hypertension, who was incidentally discovered to have an adrenal tumour suggestive of a cortical adenoma, which was later proved to be a pheochromocytoma.

\section{Case report}

A 72-year-old woman presented with a three month history of episodic vomiting and intermittent pain in the right upper quadrant of the abdomen. Her routine ultrasonography detected a 3-cm-wide mass in the right adrenal gland. CT scanning confirmed the finding and revealed a well-defined homogenous, hypodense mass lesion, measuring $3.5 \times 3.0 \mathrm{~cm}$, in the right supra renal region, with marked enhancement after contrast. There was no hyper or hypoplasia in the contralateral left adrenal gland (Figure 1).

In the past, the patient had suffered from mild hypertension, hypothyroidism, and osteopaenia of the lumbar spine and hip (spine T score -1.7, Z -0.6, hip T -2.0, $\mathrm{Z}-0.7$ ) of 2,10 and 2 years respectively. For these, she was treated with long acting calcium channel blockers, levothyroxine and raloxifene. Recently, she had experienced occasional palpitations without any episodic headache or sweating, and her blood pressure control deteriorated despite good compliance with treatment.

Her BMI was $18.3 \mathrm{~kg} / \mathrm{m}^{2}$. There were no classical signs of Cushing syndrome such as purple striae, facial plethora, dorsocervical fat pad or ecchymosis. Blood pressure was 160/ $90 \mathrm{mmHg}$, and pulse rate was 90/min. No enlarged thyroid was palpable. There were no other abnormal clinical signs.

Her baseline investigations including serum calcium, creatinine and electrolyte levels were normal. In view of incidentally detected adrenal mass, further adrenal hormone evaluation was undertaken. (normal values are

${ }^{1}$ Consultant Physician and Endocrinologist, Nawaloka Hospital, Colombo, ${ }^{2}$ Senior Registrar in Endocrinology, ${ }^{3}$ Consultant Endocrinologist, ${ }^{4}$ Consultant Histopathologist, ${ }^{5}$ Consultant Surgeon, National Hospital of Sri Lanka, Colombo, Sri Lanka. 
given in parenthesis). The morning serum cortisol level was $27 \mathrm{mcg} / \mathrm{dL}$ (4 to $30 \mathrm{mcg} / \mathrm{dL}$ ). Following administration of dexamethasone, $0.5 \mathrm{mg}$ every 6 hours for 48 hours, and thereafter 2 mg every 6 hours for 48 hours, the serum cortisol levels were $4.47(1.8 \mathrm{mcg} / \mathrm{dL})$ and $2.8 \mathrm{mcg} / \mathrm{dL}$ ( $>50 \%$ reduction) respectively. The failure of dexamethasone to suppress cortisol production in the above tests, indicated autonomous cortisol production from the adrenal gland. The serum levels of ACTH was $15 \mathrm{pg} / \mathrm{mL}$ ( 0 to $46 \mathrm{pg} / \mathrm{mL}$ ), testosterone $0.3 \mathrm{ng} / \mathrm{mL}$ ( 0.0 to $0.7 \mathrm{ng} / \mathrm{mL}), \mathrm{T} 41.23 \mathrm{ng} / \mathrm{dL}$ (0.89-1.76 ng/dL) and TSH 2.3 IU/mL ( 0.4-4 IU/mL). The plasma renin activity and aldosterone level at $8 \mathrm{am}$, after being ambulatory for 2 hours and seated for 15 minutes were $0.68 \mathrm{ng} / \mathrm{mL} / \mathrm{hr}$ ( $1-4 \mathrm{ng} / \mathrm{mL} / \mathrm{hr}$ ) and $21.87 \mathrm{ng} / \mathrm{dL}$ ( 5-30 $\mathrm{ng} / \mathrm{dL}$ ) respectively. The ARR was high at 32 (normal $<20$ ) which was suggestive of primary aldosteronism. The 24-
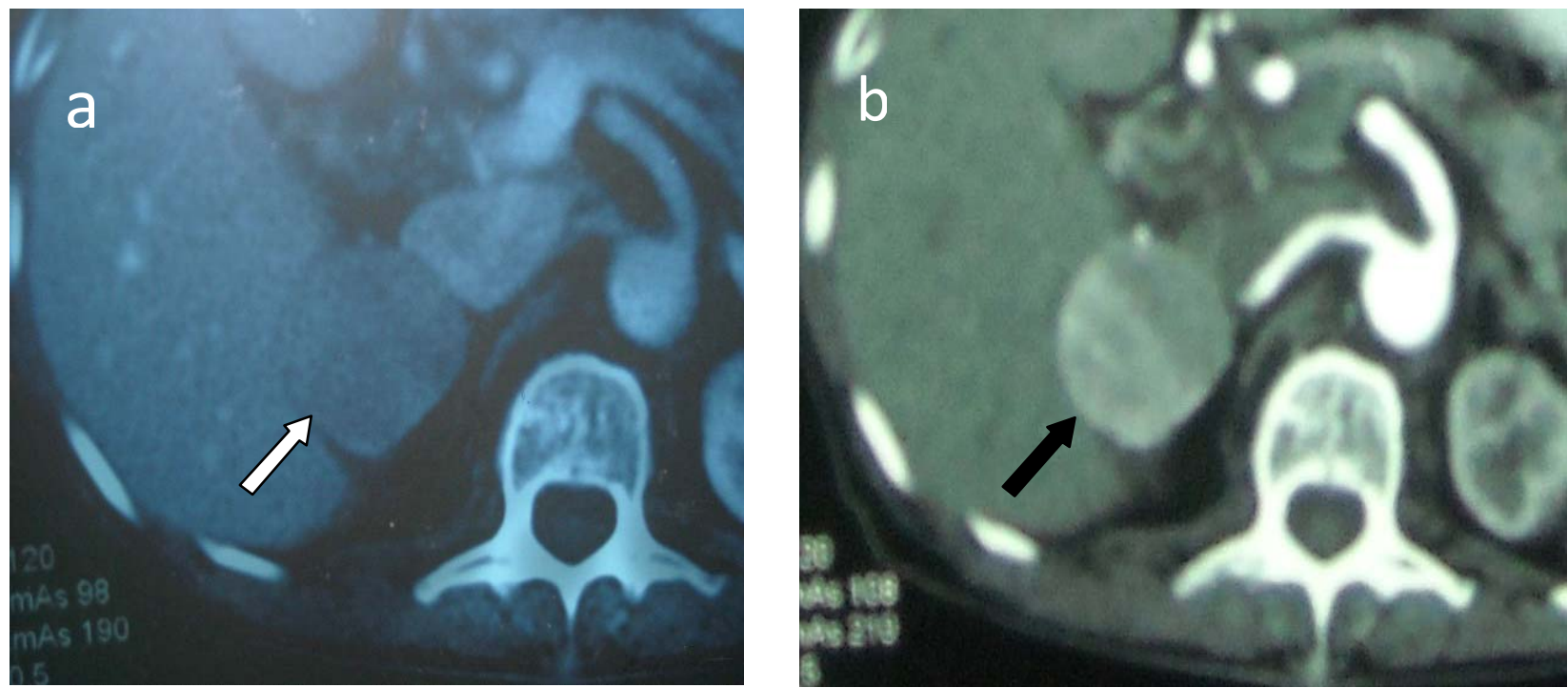

Figure 1. a) Pre-contrast CT scan showing the right sided well defined hypodense adrenal tumour (white arrow). b) Post-contrast CT image showing the contrast enhancement (black arrow).

Table 1. Fludrocortisone suppression test
Basal plasma aldosterone - upright
$41.7 \mathrm{ng} / \mathrm{dL}$
( 7-30 ng/dL)
Plasma aldosterone on day 4 of
fludrocortisone - upright
$70.1 \mathrm{ng} / \mathrm{dL}$
$(<6 \mathrm{ng} / \mathrm{dL})$ 

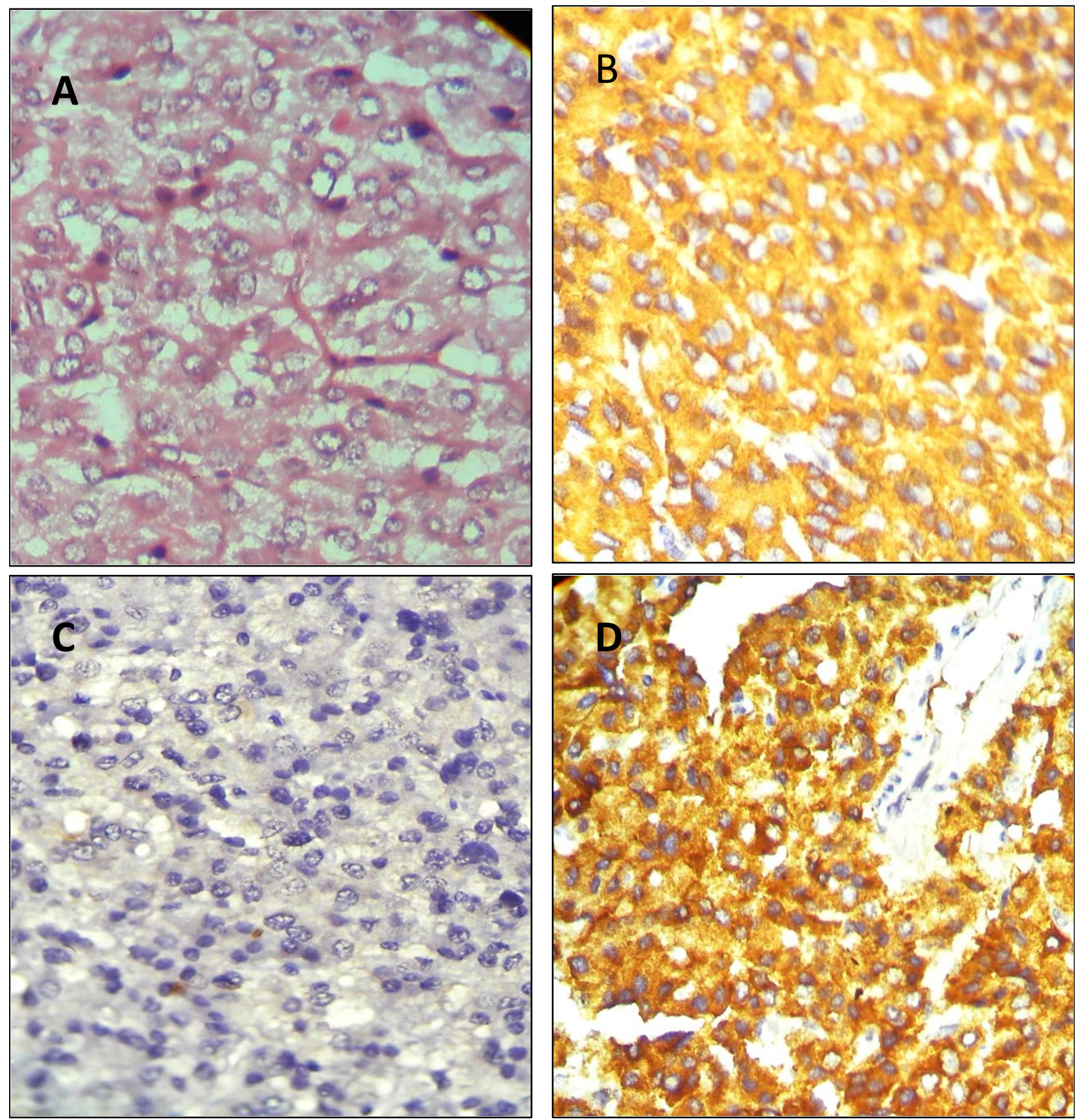

Figure 2. A) H \& E section shows tumor cells B) Chromogranin A immunostaining is diffusely positive in the tumor cells. C) Tumour cells negative for cytokeratin (CK) immunostaining. D) Synaptophysin staining shows diffuse cytoplasmic staining in the tumor cells.

Though a right adrenocortical adenoma was suspected based on conspicuous biochemical and imaging characteristics, marginally elevated urinary VMA and metanephrines mandated a consideration of a functioning pheochromocytoma (primary or coexisting) in this patient. The patient was given oral phenoxybenzamine for 10 days preoperatively, starting with $10 \mathrm{mg} 12$ hourly and gradually increasing up to $20 \mathrm{mg} 12$ hourly. With treatment, the blood pressure came down to normal levels (systolic BP 110 to $80 \mathrm{mmHg}$ and diastolic BP 80 to $60 \mathrm{mmHg}$ ). The patient was also given propanolol $10 \mathrm{mg}$ three times daily for 5 days, starting from day eleven. She underwent laparoscopic right adrenalectomy. Intra operative and post-operative periods were uneventful. Hydrocortisone replacement therapy was started to counter possible postoperative hypocortisolism, caused by suppression of the contralateral adrenal gland by the hypercortisolism produced by the autonomous adrenal tumour. She was discharged on post-operative day 5 on a single antihypertensive agent. 
One month after surgery, hydrocortisone was omitted after recording normal baseline values without replacement. The blood pressure was well controlled on prazosin $0.5 \mathrm{mg}$ t.d.s. Post-operative hormonal analysis revealed a fasting basal serum cortisol level of $12.5 \mathrm{mcg} /$ $\mathrm{dL}$, suppressed to less than $1.8 \mathrm{mcg} / \mathrm{dL}$ with the low dose dexamethasone suppression test, ARR of $16.5(<20)$ and a 24 hour urine metanephrines of $38.6 \mathrm{ng} / \mathrm{mL}$ (0-350 $\mathrm{ng} / \mathrm{mL})$. Histopathology of the right adrenal gland revealed the tumour to be a pheochromocytoma with surrounding normal adrenal tissue. Immunohistochemical staining of the tumour demonstrated cells that were strongly positive for chromogranin A, synaptophysin and S100, while it was negative for cytokeratin (CK). Surrounding adrenal tissue showed normal adrenal gland features and failed to show any other type of tumour (Figure 2).

\section{Discussion}

Pheochromocytomas and adrenocortical adenomas derive from distinct origins. Therefore, simultaneous occurrence of these two entities is extremely rare, especially in the same adrenal gland. There had only been a few cases of pheochromocytomas, either with functioning or non-functioning adrenal cortical adenoma, reported in the literature (2). At diagnosis, the patient's tumour was thought to be non-functional, because she didn't have definitive signs or symptoms of a syndrome associated with excessive levels of adrenal hormones, except for hypertension. However autonomous secretion of aldosterone, cortisol and catecholamines was established after hormonal evaluation.

Subclinical Cushing syndrome (i.e., glucocorticoid secretory autonomy without clinical manifestations of Cushing syndrome) is the most frequent hormonal abnormality detected in patients with adrenal incidentalomas (3). Because of the lack of sensitivity of most ACTH assays at the lower part of the reference range, most centers rely on an alternative measure of autonomous cortisol secretion such as the overnight dexamethasone suppression test (DST). Hyper functioning of both the medulla and cortex of an adrenal gland is more commonly seen when a pheochromocytoma secretes ACTH or corticotrophin releasing factor (CRF) ectopically. This was not the case in our patient, as she had a low normal level of ACTH and had no hyperplasia in the contra lateral adrenal gland. Non suppressed cortisol following $8 \mathrm{mg} / \mathrm{d}$ of dexamethasone for 2 days, suggested that the excess cortisol was of adrenal origin. The simultaneous hyper secretion of catecholamines, cortisol and aldosterone can be explained by many mechanisms. Catecholamines have been shown to have an effect on steroidogenesis of adjacent cortical cells in a paracrine manner (4). Human pheochromocytomas may also synthesize and secrete several regulatory peptides, such as adrenomedullin, somatostatin, neuropeptide $Y$, and galanin, which are able to influence adrenocortical steroid production. Long term secretion of ACTH, catecholamines and several other peptides might result in adrenal cortical adenoma formation (4). However, it is still difficult to explain why most patients with pheochromocytoma do not develop cortical hyperplasia or adenomas.

Pheochromocytomas are being increasingly discovered in the presymptomatic stage, due to the widespread use of CT and MRI. Approximately 3 percent of adrenal incidentalomas prove to be pheochromocytomas (5). Although in pheochromocytomas, urine metanephrines are elevated 2-3 times the upper limit of normal value, a study revealed that thirty percent of patients with adrenal incidentalomas have a final diagnosis of pheochromocytoma with urine or plasma metanephrines which were only borderline elevated (6).

Adrenocortical carcinomas have been reported to secrete multiple hormones including catecholamines (7). They are usually large at diagnosis (more than $4 \mathrm{~cm}$ ) and appear heterogeneous in images owing to the presence of internal hemorrhage and necrosis (8). These features were not seen in this patient. They have distinct histological features and immunohistochemical staining which help to differentiate them from a pheochromocytoma. Both pheochromocytoma and adrenal cortical carcinoma are immunoreactive for synaptophysin, although the staining with a pheochromocytoma is much stronger. Chromogranin A, another diffuse neuroendocrine marker, is not positive in adrenal cortical tissues, making it one of the most useful immunohistochemical markers in distinguishing adrenal medullary tumors from adrenal cortical tumors. Cytokeratins, which are positive in adrenal cortical tumors, are also helpful in distinguishing between these two entities (9). In this patient there was no evidence to suggest the presence of an adrenocortical carcinoma and immunohistochemical staining of the tumour confirmed that it was of medullary origin.

This patient had unequivocal evidence of autonomous aldosterone secretion which resolved after the tumour removal. Although the "imaging phenotype" of the tumour in the right adrenal gland (less than $4 \mathrm{~cm}$, homogenous, hypodense round mass with regular borders) was more in favour of a cortical adenoma, histology and immunohistochemical staining proved it to be a pheochromocytoma. Unfortunately, we couldn't locate the exact source of the excess aldosterone. The most possible explanation for this would be that even though careful histological analysis of the specimens was done, there may have been a small aldosterone producing cortical microadenoma, which may not have been included in the cut up specimens taken up for histology. The fact 
that patient's ARR normalized after surgery, made this the most likely explanation, than a missed small aldosterone producing adenoma in the contralateral adrenal gland. The current guidelines recommend performing adrenal venous sampling to localize the site of aldosterone producing tumour, before proceeding to surgery (10). Since adrenal incidentalomas are common, this is a safe approach in those above 40 years of age.

About 10 percent of all catecholamine-secreting tumors are malignant (11). Malignant pheochromocytomas are histologically and biochemically similar to benign ones and the only true indicator of malignant behaviour is metastatic spread. Our patient was advised to attend the follow up clinic annually, with hormonal assessment to detect any recurrence.

This case is a good example to emphasize the fact that careful and detailed endocrine evaluation is warranted in each patient with an incidentally discovered adrenal mass. With the available resources, we were able to reasonably establish that the patient's right adrenal gland had autonomous mixed hormonal secretion.

\section{References}

1. Terzolo M, Stigliano A, Chiodini I, et al. AME position statement on adrenal incidentaloma. Eur J Endocrinol 2011; 164(6): 851-70.

2. Hwang WR, Ma WY, Tso AL, Pan CC, Chang YH, Lin HD. Pheochromocytoma and adrenocortical adenoma in the same gland. J Chin Med Assoc 2007; 70(7): 289-93.

3. Emral R, Uysal AR, Asik M, et al. Prevalence of subclinical Cushing's syndrome in 70 patients with adrenal incidentaloma: clinical, biochemical and surgical outcomes. Endocr J 2003; 50(4): 399-408.

4. Ehrhart-Bornstein M, Hinson JP, Bornstein SR, Scherbaum WA, Vinson GP. Intraadrenal interactions in the regulation of adrenocortical steroidogenesis. Endocr Rev 1998; 19: 101-43.

5. Cawood TJ, Hunt PJ, O'Shea D, et al. Recommended evaluation of adrenal incidentalomas is costly, has high falsepositive rates and confers a risk of fatal cancer that is similar to the risk of the adrenal lesion becoming malignant; time for a rethink? Eur J Endocrinol 2009; 161: 513-27.

6. Lee JA, Zarnegar R, Shen WT. Adrenal incidentaloma, borderline elevations of urine or plasma metanephrine levels, and the "subclinical" pheochromocytoma. Arch Surg 2007; 142(9): 870-3.

7. Kawano S, Iwashita K, Yano S. A case of virilizing adrenocortical carcinoma in an adult female with increased urinary excretion of catecholamines. Gan No Rinsho. 1987; 33(6): 742-7.

8. Elsayes KM, Mukundan G, Narra VR. Adrenal masses: imaging features with pathologic correlation. Radiographics 2004; 24 Suppl 1: S73-86.

9. Erickson LA, Lloyd RV. Practical Markers Used in the Diagnosis of Endocrine Tumors. Adv Anat Pathol 2004; 11(4): 175-89.

10. Funder JW, et al. Case detection, diagnosis, and treatment of patients with primary aldosteronism: an Endocrine Society clinical practice guideline. J Clin Endocrinol Metab 2008; 93: 3266-81.

11. Harari A, Inabnet WB 3rd. Malignant pheochromocytoma: a review. Am J Surg 2011; 201(5): 700-8. 